3 Metze D, Rutten A. Granular parakeratosis - a unique acquired disorder of keratinization. J Cutan Pathol 1999; 26: 339-352.

4 Fujii M, Kishibe M, Honma M, Anan T, Ishida-Yamamoto A. Aluminum chloride-induced apoptosis leads to keratinization arrest and granular parakeratosis. Am J Dermatopathol 2019; https://doi.org/10. 1097/DAD.0000000000001513.

5 Webster CG, Resnik KS, Webster GF. Axillary granular parakeratosis: response to isotretinoin. J Am Acad Dermatol 1997; 37: 789-790.

\title{
Clouston syndrome with dental anomalies, micropores of hair shafts and absence of palmoplantar keratoderma
}

Dear Editor,

A 3-year-old Thai girl presented with hypotrichosis, pale and slow-growing hair. At birth she had normal hair, but she progressively lost it. Her hair stayed short. Hair loss commenced in the temporo-occipital areas (Fig. 1a). Her fingernails were narrow and hyperconvex, but toenails were normal (Fig. 1b). Scanning electron microscopy (SEM) of scalp hair showed hypoplastic cuticles (Fig. 1c). Oral examination showed

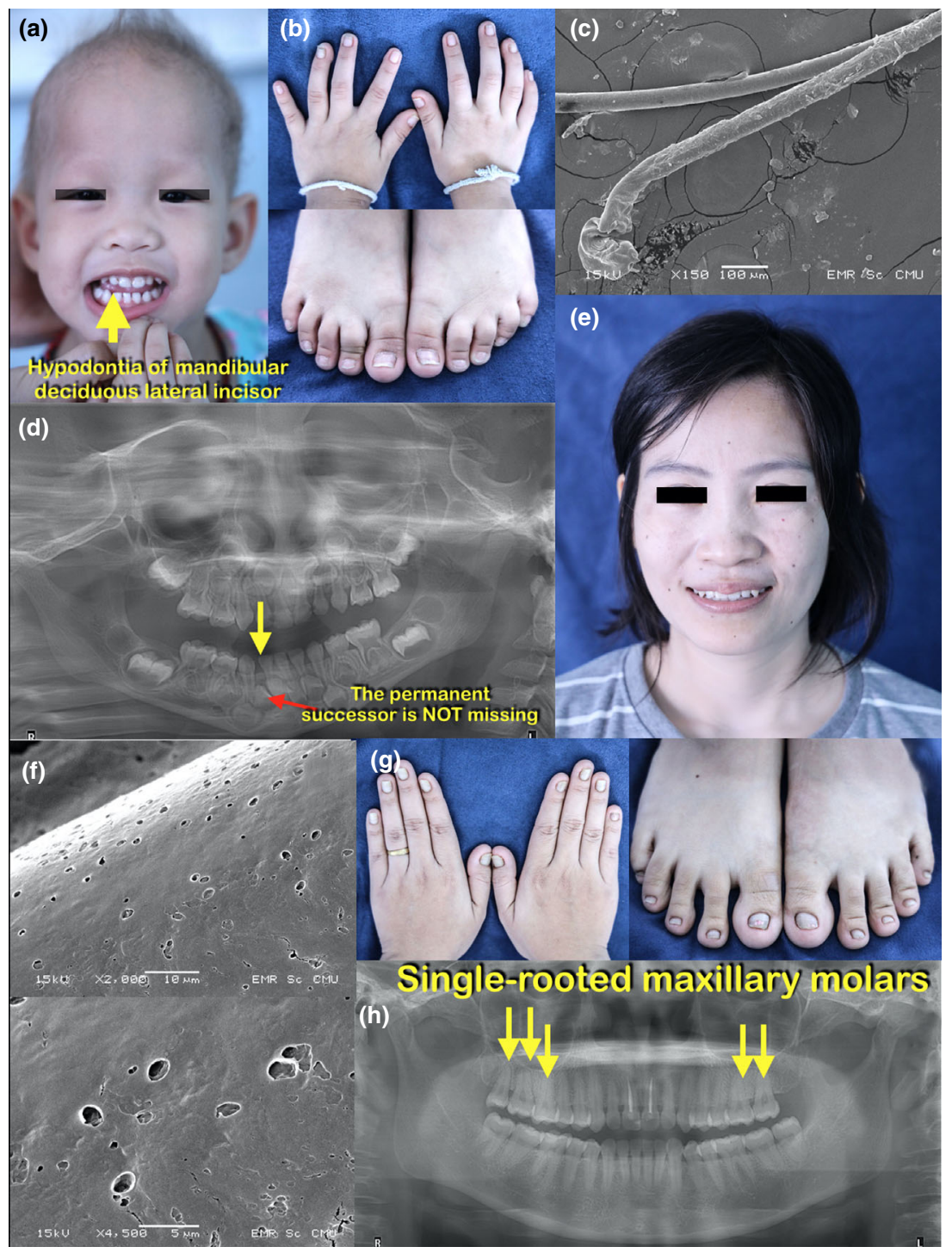

Figure 1. (a) The proband with hypotrichosis, pale and slow-growing hair. Hypodontia of the mandibular deciduous right lateral incisor (arrow). (b) Fingernails are narrow and hyperconvex, but toenails are unremarkable. (c) Scanning electron microscopy (SEM) of scalp hair shows hypoplastic cuticles. (d) Panoramic radiograph shows hypodontia of the mandibular right deciduous lateral incisor (yellow arrow). The successor of the missing deciduous tooth is observed (red arrow). (e) The mother of the proband is of normal appearance. Skin and hair appears essentially normal. (f) SEM of her scalp hair shows absence of cuticles. Note numerous micropores on the hair shafts near hair follicles. Micropores are smaller than $5 \mu \mathrm{m}$. (g) Fingernails and toenails are narrow and hyperconvex. (h) Panoramic radiograph shows single-rooted maxillary permanent molars (arrows).

Correspondence: Piranit Nik Kantaputra, D.D.S., M.S., Division of Pediatric Dentistry, Department of Orthodontics and Pediatric Dentistry, Faculty of Dentistry, Chiang Mai University, Chiang Mai 50200, Thailand. Email: dentaland17@gmail.com 
hypodontia of the mandibular right deciduous lateral incisor. Interestingly, the successor of the missing deciduous tooth was radiographically present (Fig. 1a,d). Intelligence, sweating and hearing were unremarkable. The mother had normal skin and hair (Fig. 1e). SEM of her scalp hair showed numerous micropores along the hair shafts near hair follicles (Fig. 1f). Her fingernails and toenails were narrow and hyperconvex (Fig. 1g). A panoramic radiograph showed single-rooted maxillary permanent molars (Fig. 1h).

Following approval of the ethics committee, obtaining informed consent and in full accordance with the Declaration of Helsinki, whole-exome sequencing was performed on the proband's DNA. Whole-exome sequencing showed a heterozygous single base substitution in GJB6 (c.31G>A; p.Gly11Arg) (Fig. S1) and GJB2 (c.109G>A; p.Val37lle) (Fig. S2). Sanger sequencing revealed the presence of GJB2 and GJB6 variants in the proband and her mother, but not the father.

The c.31G $>A$; p.Gly11Arg missense mutation in GJB6 has been known to be the cause of Clouston syndrome (Mendelian Inheritance in Man \#129500). It is characterized by hypotrichosis, pale, brittle and slow-growing hair, abnormal nails and palmoplantar keratoderma. Four GJB6 mutations (Gly11Arg, Val37Glu, Asp50Asn, Ala88Val) have been reported. ${ }^{1}$ The allele frequencies of the GJB2 variant according to ExAC and gnomAD are 0.00659 and 0.00772 , respectively. According to the Leiden Open Variation Database, this GJB2 variant has been reported to be pathogenic for hearing impairment in a number of reports. Single-rooted maxillary permanent molars and micropores on the scalp hair shafts found in the mother have never been reported in patients with Clouston syndrome. The micropores may be places where cuticle cells have fallen off the hairs as a result of poorly formed gap junction connections. Whole-exome sequencing excluded the effects of other known hypodontia-associated genes including WNT10A, WNT10B, AXIN2, PAX9, MSX1, GREM2 and TFAP $2 B .^{2}$ It is convincing that the dental anomalies found in this family were the effects of GJB6 mutation. The absence of a deciduous tooth with the presence of its permanent successor implies the differential role of GJB6 in development of the primary dentition, but not the permanent one. Up till now, hypodontia has only been reported in Thai patients who carried the p.Ala88Val mutation. ${ }^{1}$ Dental anomalies, micropores on the hair shafts and the absence of palmoplantar keratoderma might have been the effects of racial background or the combined effects of GJB6 and GJB2 variants. $^{3}$ The dental and hair findings raise the importance of GJB6 in tooth and hair development and this hypothesis is supported by the findings that GJB6 is a potential direct target gene of TP63 ${ }^{4}$ and dental and hair anomalies are common in patients with TP63 mutations. ${ }^{5}$ GJB2 may function as a modifier gene because GJB2 itself is not expressed in teeth during early tooth development.

ACKNOWLEDGMENTS: We thank our patients and their families for their kind cooperation and for allowing us to use their medical and dental information for the benefit of others. We thank Professor John A. McGrath for critical review of the manuscript. This work was supported by the Thailand Research Fund; the Dental Association of Thailand; and the Faculty of Dentistry, Chiang Mai University.

\section{CONFLICT OF INTEREST: None declared.}

\author{
Piranit Nik KANTAPUTRA, ${ }^{1,2}$ iD \\ Worrachet INTACHAI, ${ }^{1}$ Bruce M. CARLSON, ${ }^{3}$ \\ Chulabhorn PRUKSACHATKUNAKORN ${ }^{4}$ \\ ${ }^{1}$ Division of Pediatric Dentistry, Department of Orthodontics and Pediatric \\ Dentistry, Faculty of Dentistry, Chiang Mai University, ${ }^{2}$ Dentaland Clinic, \\ Chiang Mai, Thailand, ${ }^{3}$ Department of Anatomy and Cell Biology, \\ University of Michigan, Ann Arbor, Michigan, USA, and ${ }^{4}$ Division of \\ Pediatric Dermatology, Department of Pediatrics, Chiang Mai University, \\ Chiang Mai, Thailand \\ doi: $10.1111 / 1346-8138.15236$
}

\section{REFERENCES}

1 Sukakul T, Yang HS, Onoufriadis A, Hsu CK, McGrath JA. Pterygium and thinning of nails as an unual manifestation in Clouston syndrome. J Dermatol 2019; 46: e329-e330.

2 Kantaputra PN, Hutsadaloi A, Kaewgahya M et al. WNT10B mutations associated with isolated dental anomalies. Clin Genet 2018; 93: 992-999.

3 Sugiura K, Teranishi M, Matsumoto Y, Akiyama M. Clouston syndrome with heterozygous GJB6 mutation p.Ala88Val and GJB2 variant p.Val27lle revealing mild sensorineural hearing loss and photophobia. JAMA Dermatol 2013; 149: 1350-1351.

4 Fujimoto A, Kurban M, Nakamura M et al. GJB6, of which mutations underlie Clouston syndrome, is a potential direct target gene of p63. J Dermatol Sci 2013; 69: 159-166.

5 Kantaputra PN, Hamada T, Kumchai T, McGrath JA. Heterozygous mutation in the SAM domain of p63 underlies Rapp-Hodgkin ectodermal dysplasia. J Dent Res 2003; 82: 433-437.

\section{SUPPORTING INFORMATION}

Additional Supporting Information may be found in the online version of this article:

Figure S1. A heterozygous single base substitution in GJB6 (c.31G $>A$; p.Gly11Arg) in the patient and her mother (arrows).

Figure S2. A heterozygous single base substitution in GJB2 (c.109G $>$ A; p.Val37lle) in the patient, her mother, and a normal control (arrows). 